\title{
A hybridization of electromagnetic-like mechanism and great deluge for examination timetabling problems
}

\author{
Salwani Abdullah ${ }^{1}$, Hamza Turabieh ${ }^{1}$, and Barry McCollum ${ }^{2}$ \\ ${ }^{1}$ Center for Artificial Intelligence Technology, \\ Universiti Kebangsaan Malaysia, 43600 Bangi, Selangor, Malaysia \\ \{salwani, hamza\}@ftsm.ukm.my \\ ${ }^{2}$ Department of Computer Science, Queen's University Belfast, \\ Belfast BT7 1NN United Kingdom \\ b.mccollum@qub.ac.uk
}

\begin{abstract}
In this paper, we present a hybridization of an electromagneticlike mechanism (EM) and the great deluge (GD) algorithm. This technique can be seen as a dynamic approach as an estimated quality of a new solution and a decay rate are calculated at every iteration during the search process. These values are depending on a force value calculated using the EM approach. It is observed that applying these dynamic values help to generate high quality solutions. Experimental results on benchmark exam timetabling demonstrate the effectiveness of this hybrid EM-GD approach compared with previous available methods. Possible extensions upon this simple approach are also discussed.
\end{abstract}

Key words: Hybrid metaheuristic, Electromagnetism-like mechanism, Great deluge, Decay rate, Exam timetabling

\section{Introduction}

Examination timetabling problems are very common in schools and universities. Solutions to the problem concerns the allocating a set of exams, into a limited number of timeslots (periods), subjects to a set of constraints. Carter et al. [10] quoted that the basic challenge of examination timetabling problem is to schedule examinations over a limited set of timeslots so as to avoid conflicts and to satisfy a number of side-constraints. In this case, the conflict is referred as hard constraints and side-constraints are referred as soft constraints. Generally accepted hard constraints of examination timetabling problem are (i) there must be enough seating capacities and (ii) no student should be required to sit two examinations at the same time. Solutions that satisfy all hard constraints are often called feasible solution. Most likely soft constraints as reported in Burke et al.[5] are: students should not be scheduled to sit more than one examination in a day; students should not be scheduled to sit examinations in two consecutive timeslots; each student's examinations should be spread as evenly as possible over the schedule and examinations of the same length may be scheduled in the 
same room. A particularly common soft constraint refers to spreading exams as evenly as possible over the schedule. In real world situation, it is, of course, usually impossible to satisfy completely all soft constraints. Therefore efforts are made in minimizing these violations to increase the quality of the solution by calculating the penalty function to the extent to which a timetable has violated its soft constraints. McCollum et al.[18,17] introduced a new formulation of the problem as part of ITC2007. This more comprehensive description of the problem describes a range of hard and soft constraints found in recent practical problems from within the literature and practical experience. The intention here is to show the effectiveness of our technique on the original Carter datasets (see Carter et al.[12], Burke et al.[6]) before continuing the applications to this recently introduced formulation.

In the past, a wide variety of approaches for solving the examination timetable problem have been described and discussed in the literature that can be categorized into: sequential methods, cluster methods, constraint-based methods, generalised search (meta-heuristics), multi-criteria approaches, case based reasoning techniques, and hyper-heuristics/self adaptive approaches (Carter and Laporte [11], Petrovic and Burke [20]). For a recent detailed overview readers should consult $\mathrm{Qu}$ et al [22]. These approaches are tested on various examination timetabling datasets that can be found from http://www.asap.cs.nott. ac.uk/resource/data. Interested readers can find more details about examination timetabling research in the comprehensive survey paper by $\mathrm{Qu}$ et al.[22] and Lewis [16].

The remainder of this paper is as follow; Section 2 provides a review on available hybrid approaches applied on examination timetabling problems; Section 3 provides the necessary information on the formulation of the examination timetabling problem; Section 4 describes the detailed implementation of the electromagnetic metaheuristic along with the neighbourhood structures used. The simulation results are presented in Section 5. Finally, the paper is concluded making comment on the effectiveness of the technique studied and potential future research area.

\section{Hybrid Approach: Research and Developments}

A hybrid approach subsumes two or more methods. The advantage of combining several methods is that it helps to compensate for the insufficiency of using each type of method in isolation.

Burke et al.[5] developed a memetic algorithm where light and heavy mutations were employed. Hill climbing was used to improve the quality of timetables. The approach was tested on Nottingham dataset (see Qu et al.[22]). Merlot et al.[19] employed constraint programming to produce initial solutions. A simulated annealing approach is used to improve the solution. Subsequently a hill climbing method is employed to further improve the quality of the solutions. The overall hybrid approach was tested on the Carter, University of Melburne and Nottingham datasets. For details on examination timetabling benchmark data, 
please refer to $\mathrm{Qu}$ et al. [22]. This approach obtained the best results reported in the literature on several instances at the time. Casey and Thompson [13] investigated a greedy randomized adaptive search procedure (GRASP) approach where the initial solution was generated by a modified saturation degree heuristic. Backtracking was employed in conjunction with a tabu list. A simulated annealing approach (with kempe chain moves) with high starting temperature and fast cooling was used in the improvement phase. The approach was applied on Carter's dataset and able to obtain competitive results at the time. Burke et al. [7] investigated a knowledge based technique i.e. case-based reasoning as a heuristic selector and tested on four examination datasets. Different ways of hybridizing the low level graph heuristics were compared for solving Carter's datasets and was able to produce good results. Yang and Petrovic [23] employed case-based reasoning to choose graph heuristic to construct initial solutions for the great deluge algorithm. The approach obtained the best results reported in the literature for several Carter's datasets. Abdullah and Burke[1] investigated a hybridization of the very large neighbourhood search approach with local search methods to address examination timetabling problems. The approach was treated as a two phase approach where a very large neighbourhood of solutions using graph theoretical algorithms was implemented in a first phase. The second phase made further improvement by utilizing local search methods (simulated annealing and great deluge individually). Other related works on hybrid approaches applied on university timetabling problems can be found in Côtè et al.[14] that applied a hybrid multi-objective evolutionary algorithm for the exam timetabling problem, Qu and Burke [21] who investigated the hybridization within a graph based hyper-heuristic for university timetabling problems, Qu et al.[22] employed a dynamic hybridisation of different graph colouring heuristics, and Burke et al.[8] employed hybrid variable neighbourhood search for the same instances.

\section{Problem Description}

The problem description that is employed in this paper is adapted from the description presented in Burke et al. [6]. The input for the examination timetabling problem can be stated as follows:

- $E_{i}$ is a collection of $\mathrm{N}$ examinations $(i=1, \ldots, N)$.

$-T$ is the number of timeslots.

- $C=\left(c_{i j}\right)_{N \times N}$ is the conflict matrix where each record, denoted by $c_{i j}(i, j \in$ $\{1, \ldots, N\})$, represents the number of students taking exams $i$ and $j$.

- $M$ is the number of students.

$-t_{k}\left(1 \leq t_{k} \leq T\right)$ specifies the assigned timeslots for exam $k(k \in\{1, \ldots, N\})$.

The following hard constraint is considered based on Carter et al. [12]: no students should be required to sit two examinations simultaneously. 
In this problem, we formulate an objective function which tries to spread out exams throughout the exam period (Expression (1)).

$$
\text { Min } \frac{\sum_{i=1}^{N-1} F(i)}{M}
$$

where:

$$
\begin{gathered}
F(i)=\sum_{j=i+1}^{N} c_{i j} \cdot \operatorname{proximity}\left(t_{i}, t_{j}\right) \\
\operatorname{proximity}\left(t_{i}, t_{j}\right)= \begin{cases}2^{5} / 2^{\left|t_{i}-t_{j}\right|} & \text { if } 1 \leq\left|t_{i}-t_{j}\right| \leq 5 \\
0 & \text { otherwise }\end{cases}
\end{gathered}
$$

subject to:

$$
\sum_{i=1}^{N-1} \sum_{j=i+1}^{N} c_{i j} \cdot \lambda\left(t_{i}, t_{j}\right)=0 \quad \text { where }: \quad \lambda\left(t_{i}, t_{j}\right)= \begin{cases}1 & \text { if } t_{i}=t_{j} \\ 0 & \text { otherwise }\end{cases}
$$

Equation (2) presents the cost for an exam i which is given by the proximity value multiplied by the number of students in conflict. Equation (3) represents a proximity value between two exams (Carter et al.[12]). Equation (4) represents a clash-free requirement so that no student is asked to sit two exams at the same time. The clash-free requirement is considered as a hard constraint.

\section{A Hybridisation of Electromagnetism-like Mechanism and Great Deluge Algorithm}

\subsection{Solution Representation}

A direct representation is used. Each population member is represented as a number of genes that contain information about the timeslot and exams. Fig.1 shows examples of the genes where $t_{j}$ is a timeslot $(j \in\{1, \ldots, T\}), e_{i}$ is an exam $(i \in\{1, \ldots, N\})$. For example; $e_{2}, e_{11}, e_{8}, e_{7}, e_{14}$ are allocated in timeslot $t_{1}$, while $e_{21}$, and $e_{19}$ allocated in $t_{4}$.

\subsection{Electromagnetic-like Mechanism}

Birbil and Fang [3] constructed the electromagnetic-like mechanism drawing upon the attraction-repulsion mechanism of the theory of electromagnetism. Each sample point (timetable in this case) is released to a space as a charged particle whose charge relates to the objective function value. The charge determines the magnitude of attraction or repulsion of the point over the sample population. The better the objective function value, the higher the magnitude of attraction. The attraction directs the points toward better regions, whereas repulsion allows particles to exploit the unvisited regions. In this paper, the electromagnetismlike mechanism starts with a population of randomly generated timetables. The 


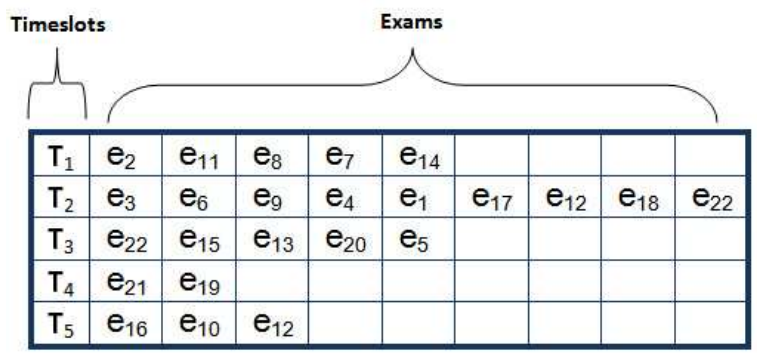

Fig. 1. Solution representation

static force between two point charges is directly proportional to the magnitudes of each charge and inversely proportional to the square of the distance between the charges (see Birbil and Fang [3]). In this paper, the fixed charge of timetable $i$ is calculated as follows:

$$
q^{i}=\exp \left(-T \frac{f\left(x^{i}\right)-f\left(x^{b}\right)}{\sum_{k=1}^{m}\left(f\left(x^{k}\right)-f\left(x^{b}\right)\right)}\right)
$$

where:

$q^{i}$ : the charge for timetable $i$.

$f\left(x^{i}\right)$ : penalty of timetable $i$.

$f\left(x^{k}\right)$ : penalty of timetable $k$.

$f\left(x^{b}\right)$ : penalty of timetable $b$ ( $b=$ best timetable through population).

$m$ : population size.

$T$ : number of timeslots.

The solution quality or charge of each timetable determines the magnitude of an attraction and repulsion effect in the population. A better solution encourages other particles to converge to attractive valleys while a bad solution discourages particles to move toward this region. These particles move along with the total force and so, diversified solutions are generated. The following formulation is the total force of particle $i$.

$$
F_{i j}=\sum_{j \neq i}^{m}\left\{\begin{array}{lll}
\left(f\left(x^{j}\right)-f\left(x^{i}\right)\right) \frac{q^{i} q^{j}}{\left\|f\left(x^{j}\right)-f\left(x^{i}\right)\right\|^{2}} & \text { if } & f\left(x^{j}\right)<f\left(x^{i}\right) \\
\left(f\left(x^{i}\right)-f\left(x^{j}\right)\right) \frac{q^{i} q^{j}}{\left\|f\left(x^{j}\right)-f\left(x^{i}\right)\right\|^{2}} & \text { if } & f\left(x^{j}\right) \geq f\left(x^{i}\right)
\end{array}\right\}, \forall i
$$

In general, the process of evaluating the total force is illustrated in Fig.2. Three particles (labeled as 1, 2 and 3) which represent feasible solutions with their associated objective function values i.e. 30,8 and 19, respectively. Since particle 1 is worst than particle 3 , a repulsive force $F_{13}$ effects on particle 3 . Particle 2 is better than particle 3 , thus an attractive force $F_{23}$ effects on particle 
3 to force attraction-repulsive in different directions, i.e. particle 3 moves along with total force $F$.

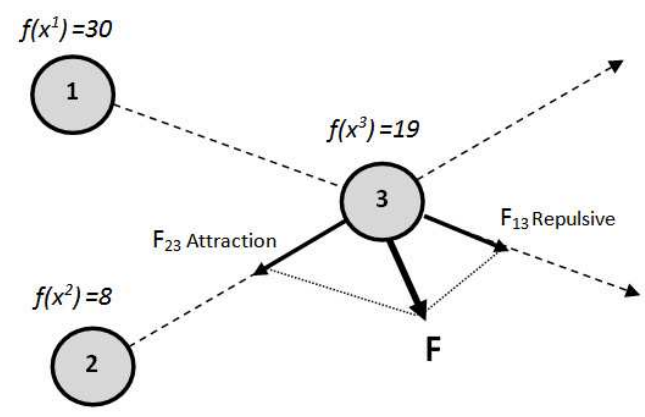

Fig. 2. Attraction- repulsive effect on particle 3

\subsection{Great Deluge Algorithm}

The great deluge algorithm was introduced by Dueck [15]. It is a local search procedure which has certain similarities with simulated annealing. This approach is far less dependent upon parameters than simulated annealing. It needs just two parameters: the amount of computational time that the user wishes to "spend" and an estimate of the quality of solution that a user requires. Apart from accepting a move that improves the solution quality, the great deluge algorithm also accepts a worse solution if the quality of the solution is less (for the case or minimisation) than or equal to some given upper boundary value $\beta$ (in the paper by Dueck it was called a "level"). In this work, the "level" is initially set to be the objective function value of the initial solution. During its run, the "level" is iteratively lowered by a constant where is a force decay rate (see Fig.3). The great deluge algorithm will be applied on each timetable to reduce the total penalty cost based on the calculated force value.

The pseudo code for the great deluge is presented in Fig.3. In this work, two types of neighbourhood structures have been applied i.e.:

N1: Select two exams at random and swap timeslots.

N2: Choose a single exam at random and move to a new random feasible timeslots.

\subsection{A Hybrid Approach}

The discussion on the hybrid approach is divided into two parts i.e. (1) Initialisation and (2) electromagnetic-like mechanism and great deluge algorithm as shown in Fig.4. In Step 1, the quality of the initial and best solutions are 
Calculate estimated quality of every solution, EstimatedQuality $=f\left(\mathrm{Sol}_{i}\right)-F_{i}$, where $i=1$ to population size;

Calculate force decay rate, $\beta=$ EstimatedQuality/NumOfite;

Define neighbourhood $\left(N_{1}\right.$ and $\left.N_{2}\right)$ of $S_{\text {Sol }}$ by randomly assigning exam to a valid timeslot to generate a new solution called Sol $_{i}^{*}$;

Calculate $f\left(\mathrm{Sol}_{i}^{*}\right)$;

if $\left(f\left(\mathrm{Sol}_{i}^{*}\right)<f\left(\mathrm{Sol}_{\text {best }}\right)\right)$ where Sol $\mathrm{l}_{\text {best }}$ represent the best solution found so far

$\mathrm{Sol}_{i} \leftarrow \mathrm{Sol}_{i}^{*} ;$

$\mathrm{Sol}_{\text {best }} \leftarrow \mathrm{Sol}_{i}^{*} ;$

else

if $\left(f\left(\mathrm{Sol}_{i}^{*}\right) \leq\right.$ level)

$\mathrm{Sol}_{i} \leftarrow \mathrm{Sol}_{i}^{*} ;$

level $=$ level $-\beta$;

Increase iteration by 1 ;

Fig. 3. Pseudo code for the great deluge algorithm

Step 1: Initialization:

Set initial solution as Sol;

Calculate the initial penalty cost, $f($ Sol);

Set best solution, Sol $\leftarrow$ Sol;

Set total number of iterations, NumOfite;

Set initial level: level $\leftarrow f($ Sol);

Set iteration $\leftarrow 0$;

Step 2: Evaluation:

do while (iteration < NumOfite)

Calculate total force, $F$, for each timetable based on

electromagnetic-like mechanism;

Apply great deluge algorithm (see Figure 3)

end do

Fig. 4. Pseudo-code for the hybrid approach 
calculated and set together with the number of iteration and level. In Step 2, electromagnetic-like mechanism is implemented to calculate the force for each solution. The force value later will be used in the great deluge algorithm to calculate the decreasing rate (in this paper, we referred as a force decay rate) as shown in Fig.4.

Fig.5 illustrates an example of seven solutions with the current objective function values (penalty). For each solution, firstly, the charge and force have to be calculated and evaluated using equations (5) and (6) respectively. For example, the penalty, charge and force for $S_{o l}$ are 175.2292, 0.1161401 and 0.0769340 , respectively. $S_{0 l}$ has a charge value $=1$ and force $=0$. This means that the quality of this timetable (particle) is no need to be reduced in the next iteration. However, for the rest of the solutions, the penalties are tried to be lowered at least at (penalty - force). Taking $S_{\text {ol }} l_{1}$ as an example, the force (F) is 0.0769340 , thus the estimated quality of the solution,Estimated Quality, (see Fig.3) will be 175.1522 (i.e. 175.2292 -0.0769340). However, the great deluge algorithm is able to reduce the penalty for each timetable less than the estimated quality. For example, after applying the great deluge, the quality of $S_{0} l_{1}$ is 174.4521 (which is less than 175.1522). Note that, this example is taken from our experiment on sta-f-83 dataset.

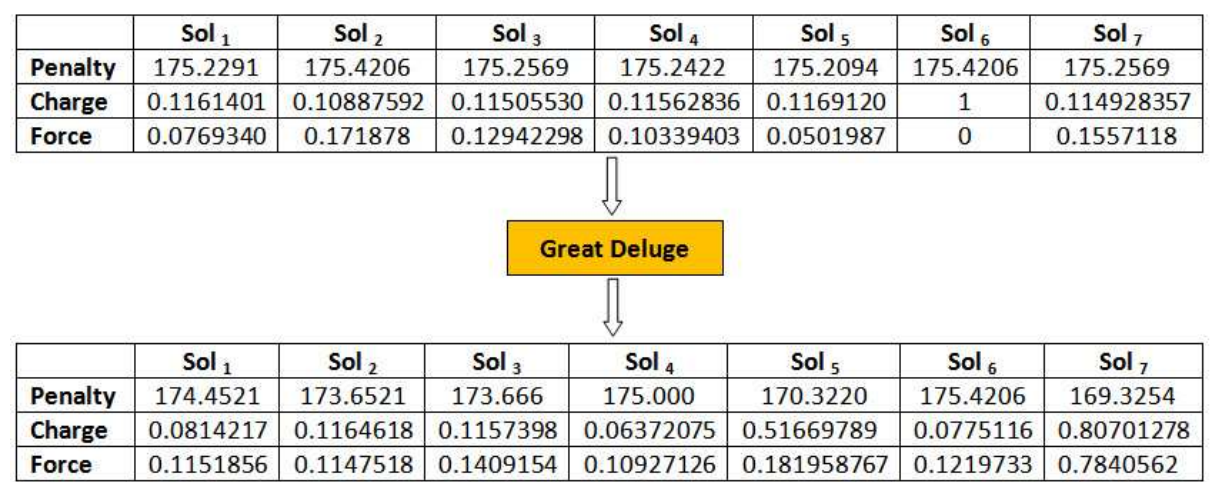

Fig. 5. Illustrative example for sta-f- 83 with seven solutions

\section{Simulation Results}

The proposed algorithm was programmed using Matlab and simulations were performed on the Intel Pentium $42.33 \mathrm{GHz}$. In this paper, we considered a standard benchmark examination timetabling problem from Carter et al. [12]. Table 1 shows the parameter for the hybrid algorithm after some preliminary experiments. 
Table 1. Parameter Setting

\begin{tabular}{cc}
\hline Parameter & Value \\
\hline Generation number & 10000 \\
Population size & 50 \\
\hline
\end{tabular}

Table 2 provides the comparison of our results with the best known results for these benchmark datasets (taken from Qu et al. [22]). The best results out of 5 runs are shown in bold.

Table 2. Comparison Results

\begin{tabular}{cccc}
\hline Instance & $\begin{array}{c}\text { Our } \\
\text { approach }\end{array}$ & $\begin{array}{c}\text { Best } \\
\text { Known }\end{array}$ & Authors for best known \\
\hline car-s-91 & $\mathbf{4 . 4 6}$ & 4.50 & Yang and Petrovic [23] \\
car-f-92 & $\mathbf{3 . 7 6}$ & 3.98 & Yang and Petrovic [23] \\
ear-f-83 & 32.12 & $\mathbf{2 9 . 3}$ & Caramia et al. [9] \\
hec-s-92 & 9.72 & $\mathbf{9 . 2}$ & Caramia et al. [9] \\
kfu-s-93 & $\mathbf{1 2 . 6 2}$ & 13.0 & Burke et al. [4] \\
lse-f-91 & 10.03 & $\mathbf{9 . 6}$ & Caramia et al. [9] \\
sta-f-83 & $\mathbf{1 5 6 . 9 4}$ & 157.2 & Côtè et al. [14] \\
tre-s-92 & $\mathbf{7 . 8 6}$ & 7.9 & Burke et al. [5] \\
uta-s-92 & $\mathbf{2 . 9 9}$ & 3.14 & Yang and Petrovic [23] \\
ute-s-92 & 24.9 & $\mathbf{2 4 . 4}$ & Caramia et al. [9] \\
yor-f-83 & $\mathbf{3 4 . 9 5}$ & 36.2 & Caramia et al. [9], Abdullah et al.[2] \\
\hline & & &
\end{tabular}

Our algorithm produces better results on seven out of eleven datasets. We are particularly interested to compare our results with the other results in the literature that employed a hybrid approach i.e.: Merlot et al. [19] that employed constraint programming as initialization for simulated annealing and hill climbing; Casey and Thompson [13] that applied GRASP with modified saturation degree initialization and simulated annealing; Yang and Petrovic [23] that employed fuzzy set on selecting hybridizations of great deluge and graph heuristics; Abdullah and Burke [1] that employed large neighbourhood search approach with local search methods, Qu and Burke [21] that investigated the hybridization within a graph based hyper-heuristic; Qu et al.[22] that employed a dynamic hybridisation of different graph colouring heuristics; and Burke et al.[8] that investigated a hybrid variable neighbourhood search.

Table 3 shows the comparison results on the hybrid algorithms as mentioned earlier. Again, the best results out of 5 runs are shown in bold. Note that the value marked "-" indicates that the corresponding problem is not tested.

From Table 3, we can see that our algorithm produces better results on almost all datasets (accept on sta-f- 83 and yor-f- 83 datasets) when compared against other hybridization methods. Note that Casey and Thompson[13] used 
Table 3. Comparison Results On Hybrid Approaches

\begin{tabular}{c|cc|ccc}
\hline & $\begin{array}{c}\text { Our } \\
\text { Instance }\end{array}$ & $\begin{array}{c}\text { Approach } \\
\text { best }\end{array}$ & \multicolumn{3}{|c}{ Merlot Casey and } \\
& & & $\begin{array}{c}\text { Côtè et } \\
\text { et al. } \\
(2003)\end{array}$ & $\begin{array}{c}\text { Thompson } \\
\text { al. }\end{array}$ \\
& & & 5.1 & 5.4 & $(2005)$ \\
\hline car-s-91 & $\mathbf{4 . 4 2}$ & 4.81 & 4.3 & 4.2 & 4.2 \\
car-f-92 & $\mathbf{3 . 7 6}$ & 3.95 & 35.1 & 34.2 & 34.2 \\
ear-f-83 & $\mathbf{3 2 . 1 2}$ & 33.69 & 10.6 & 10.2 & 10.2 \\
hec-s-92 & $\mathbf{9 . 7 3}$ & 10.10 & 10.2 & 14.2 \\
kfu-s-93 & $\mathbf{1 2 . 6 2}$ & 12.97 & 13.5 & 14.2 & 11.2 \\
lse-f-91 & $\mathbf{1 0 . 0 3}$ & 10.34 & 10.5 & 14.2 & 157.2 \\
sta-f-83 & $\mathbf{1 5 6 . 9 4}$ & 157.30 & 157.3 & 134.9 & 8.2 \\
tre-s-92 & $\mathbf{7 . 8 6}$ & 8.2 & 8.4 & 8.2 & 3.2 \\
uta-s-92 & $\mathbf{2 . 9 9}$ & 3.32 & 3.5 & - & 25.2 \\
ute-s-92 & $\mathbf{2 4 . 9}$ & 25.41 & 25.1 & 25.2 & 25.2 \\
yor-f-83 & 34.95 & 36.27 & 37.4 & 37.2 & 36.2 \\
\hline
\end{tabular}

\begin{tabular}{|c|c|c|c|c|c|}
\hline Instance & $\begin{array}{c}\text { Abdullah } \\
\text { and Burke } \\
(2006)\end{array}$ & $\begin{array}{c}\text { Yang and } \\
\text { Petrovic } \\
(2005)\end{array}$ & $\begin{array}{l}\mathrm{Qu} \text { and } \\
\text { Burke } \\
(2008)\end{array}$ & $\begin{array}{l}\text { Qu et } \\
\text { al. } \\
(2009)\end{array}$ & $\begin{array}{c}\text { Burke et } \\
\text { al. } \\
(2006)\end{array}$ \\
\hline car-s-91 & 4.1 & 4.50 & 5.16 & 5.11 & 4.6 \\
\hline car-f-92 & 4.8 & 3.93 & 4.16 & 4.32 & 3.9 \\
\hline ear-f-83 & 36.0 & 33.70 & 35.86 & 35.56 & 32.8 \\
\hline hec-s-92 & 10.8 & 10.83 & 11.94 & 11.62 & 10.0 \\
\hline kfu-s-93 & 15.2 & 13.82 & 14.79 & 15.18 & 13.0 \\
\hline lse-f-91 & 11.9 & 10.35 & 11.15 & 11.32 & 10.0 \\
\hline sta-f-83 & 159.0 & 158.35 & 159 & 158.88 & 156.9 \\
\hline tre-s-92 & 8.5 & 7.92 & 8.6 & 8.52 & 7.9 \\
\hline uta-s-92 & 3.6 & 3.14 & 3.59 & 3.21 & 3.2 \\
\hline ute-s-92 & 26.0 & 25.39 & 28.3 & 28 & 24.8 \\
\hline yor-f-83 & 36.2 & 36.35 & 41.81 & 40.71 & 34.9 \\
\hline
\end{tabular}

different version of datasets (denoted in italic). It is clearly shown that our hybrid approach out performs other hybrid approaches on all of the instances. We believe that with the help of a force decay rate generated from electromagnetismlike mechanism to determine the estimated quality and then being used as a level in the great deluge manage to reduce the penalty cost of the solution. Furthermore, in our case we applied this improvement hybrid algorithm to all the population rather than a single population. This implementation helps the whole population to converge together because only population that is better than the best solution so far or better than the level will be added into the population pool to be used in the next iteration.

Fig.6 shows the convergence of the hybrid approach when applied on staf- 83 dataset. The $\mathrm{x}$ dimension represents the number of iteration while the $\mathrm{y}$ dimension represents the penalty cost. Each point on the graph represents the 


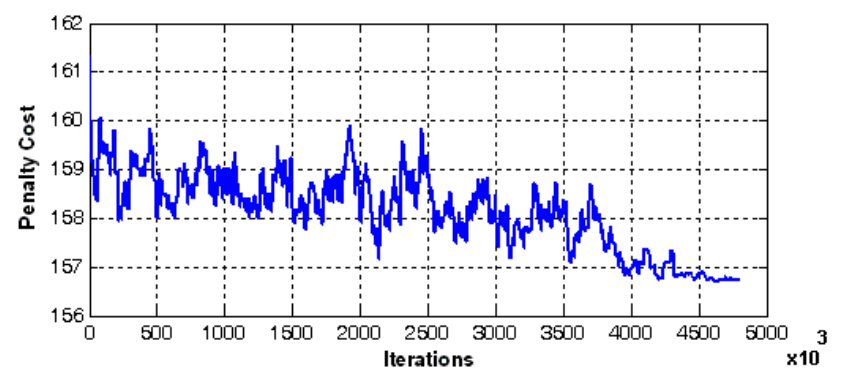

Fig. 6. Sta-f- 83 convergence using hybrid approach

solution found in each iteration. The curve up and down from the beginning until it becomes stagnant to the end of the search. This algorithm will always accepts best solutions and worse solutions will be accepted as long their quality is better than the level. This is probably because there are more rooms for improvement at the beginning of the search and towards the end of the search process less improvement is achieved. We believe that this algorithm is able to produce some of best known solutions because of the amount of the decreasing rate (based on the force value) that is calculated at every iteration is very small (see Fig.6), thus the level to be decreased is also at the very small amount. This helps the algorithm to easily accept the solutions with small improvement with respect to the objective function. It is also believed that better solutions can be obtained in these experiments with the help of a "dynamic" decreasing rate (we called "dynamic" because the value is recalculated at every iteration) where the level will be decreased based on different values (note that the decreasing rate in a standard great deluge is a predefined constant). Also, it is due to the ability of the algorithm in exploring different region of the solution space in which our algorithm works on 50 different solutions at every iteration.

\section{Conclusion and Future Work}

This paper presents a hybridization approach that combined an electromagnetismlike mechanism with great deluge algorithm. To our knowledge, this is the first such algorithm aimed at this problem domain. In order to test the performance of our approach, experiments are carried out based on examination timetabling problems and compare with a set of state-of-the-art methods from the literature. This approach is simple yet effective, and produced a number of best known results in comparison with other approaches studied in the literature. With the help of the dynamic decreasing rate that works on a set of different solutions, our approach is capable in finding better solutions for the examination timetabling problem. With the increasing complexity of examination timetabling problems in many educational institutions, we believed that the proposed effective hybrid 
approach can be adapted with new constraints easily. This is subject to our future work.

\section{References}

1. S. Abdullah,E.K. Burke: A Multi-start large neighbourhood search approach with local search methods for examination timetabling. International Conference on $\mathrm{Au}-$ tomated Planning and Scheduling (ICAPS 2006),Cumbria, UK, pp.334-337. (2006)

2. S. Abdullah, S. Ahmadi, E.K. Burke and M. Dror: Investigating Ahuja-Orlin's large neighbourhood search approach for examination timetabling. OR Spectrum, 29(2), pp.351-372 (2007)

3. S.I. Birbil, S.C. Fang: An electromagnetism-like mechanism for global optimization. Journal of Global Optimization 25,pp.263-282 (2003)

4. E.K. Burke, D.G. Elliman, P.H. Ford and R.F. Weare: Examination timetabling in British universities - A survey. The Practice and Theory of Automated Timetabling I: Selected Papers from 1st International Conference on the Practice and Theory of Automated Timetabling (PATAT I), Edinburgh, UK, LNCS 1153, Springer-Verlag, pp.76-92 (1996)

5. E.K. Burke, J.P. Newall and R.F. Weare.: A memetic algorithm for university exam timetabling. The Practice and Theory of Automated Timetabling I: Selected Papers from 1st International Conference on the Practice and Theory of Automated Timetabling (PATAT I), Edinburgh, UK, LNCS 1153, Springer-Verlag, pp.241-250 (1996)

6. E.K. Burke, Y. Bykov, J.P. Newall and S. Petrovic: A time-predefined local search approach to exam timetabling problem. IIE Transactions, 36(6), pp.509-528 (2004)

7. E.K. Burke, M. Dror, S. Petrovic and R. Qu: Hybrid graph heuristics in hyperheuristic applied to exam timetabling problems. In. B.L. Golden, S. Raghavan and E.A. Wasil (eds). The next wave in computing, optimization and decision technologies. Springer, Maryland, pp. 79-91 (2005)

8. E.K. Burke, A.J. Eckersley, B. McCollum, S. Petrovic and R. Qu: Hybrid variable neighbourhood approaches to university exam timetabling. Technical Report NOTTCS-TR-2006-2, School of Computer Science and Information Technology, University of Nottingham, United Kingdom. (2006)

9. M. Caramia, P. Dell'Olmo and G.F. Italiano: New algorithms for examination timetabling. Algorithms Engineering 4th International Workshop, Proceedings WAE 2001, Saarbrcken, Germany, LNSC 1982, Springer-Verlag, 230-241 (2001)

10. M.W. Carter, G. Laporte and J.W. Chinneck: A general examination scheduling system. Interfaces, 24(3), pp.109-120 (1994)

11. M.W. Carter and G. Laporte: Recent developments in practical examination timetabling. The Practice and Theory of Automated Timetabling I: Selected Papers from 1st International Conference on the Practice and Theory of Automated Timetabling (PATAT I), Edinburgh, UK, LNCS 1153, Springer-Verlag, pp.3-21 (1996)

12. M.W. Carter, G. Laporte and S. Lee: Examination timetabling: Algorithmic strategies and applications. Journal of the Operational Research Society, 47(3), pp.373-383 (1996)

13. S. Casey and J. Thompson: GRASPing the examination scheduling problem. The Practice and Theory of Automated Timetabling IV: Selected Papers from 4th International Conference on the Practice and Theory of Automated Timetabling (PATAT IV), Gent, Belgium, LNCS 2740, Springer-Verlag, pp.232-244 (2003) 
14. P. Côtè, T. Wong and R. Sabourin: A hybrid multi-objective evolutionary algorithm for the uncapacitated exam proximity problem. In: E.K. Burke and M. Trick (eds). Selected Papers from the 5th International Conference on the Practice and Theory of Automated Timetabling. Springer Lecture Notes in Computer Science, vol. 3616. pp.294-312 (2005)

15. G. Dueck. New optimisation heuristics: The great deluge algorithm and the recordto-record travel. Journal of Computational Physics, 104, pp.86-92 (1993)

16. R. Lewis: A survey of metaheuristic-based techniques for university timetabling problems, OR Spectrum 30 (1), pp.167-190 (2008)

17. B. McCollum, A. Schaerf, B. Paechter, P. McMullan, R. Lewis, A. Parkes, L. Di Gaspero, R. Qu, E.K. Burke: Setting the research agenda in automated timetabling: The second international timetabling competition, Accepted for publication to INFORMS Journal of Computing (2009)

18. B. McCollum, P. McMullan, E.K. Burke, A.J. Parkes, R. Qu: A New Model for Automated Examination Timetabling. Accepted to Annals of OR. Post Procedings of PATAT07, Montreal (2007)

19. L.T.G. Merlot, N. Boland, B.D. Hughes and P.J. Stuckey: A hybrid algorithm for the examination timetabling problem. In E.K. Burke and P. De Causmaecker (eds). Selected Papers from 4th International Conference on the Practice and Theory of Automated Timetabling. Springer Lecture Notes in Computer Science, vol. 2740, pp.207-231(2003)

20. S. Petrovic and E.K. Burke: University timetabling. Handbook of Scheduling: Algorithms, Models and Performance Analysis, Chapter 45, (Editor: J. Leung), CRC Press, (2004)

21. R. Qu and E.K. Burke: IHybridisations within a Graph Based Hyper-heuristic Framework for University Timetabling Problems. to appear at Journal of Operational Research Society (JORS). doi: 10.1057/jors.2008.102. (2008)

22. R. Qu, E.K. Burke and B.McCollum: Adaptive Automated Construction of Hybrid Heuristics for Exam Timetabling and Graph Colouring Problems. European Journal of Operational Research (EJOR), 198(2), 392-404 (2009)

23. Y. Yang and S. Petrovic: A novel similarity measure for heuristic selection in examination timetabling. The Practice and Theory of Automated Timetabling V: Selected Papers from 5th International Conference on the Practice and Theory of Automated Timetabling (PATAT V), Pittsburg, USA, LNCS 3616, Springer-Verlag. pp.247-269 (2005) 